\title{
Stringent comparative sequence analysis reveals SOX10 as a putative inhibitor of glial cell differentiation
}

\author{
Chetna Gopinath ${ }^{1 \dagger}$, William D. Law ${ }^{2 \dagger}$, José F. Rodríguez-Molina ${ }^{3}$, Arjun B. Prasad ${ }^{4}$, Lingyun Song ${ }^{5}$, \\ Gregory E. Crawford ${ }^{5,6}$, James C. Mullikin ${ }^{4}$, John Svaren ${ }^{7,8}$ and Anthony Antonellis ${ }^{1,2,9^{*}}$
}

\begin{abstract}
Background: The transcription factor SOX10 is essential for all stages of Schwann cell development including myelination. SOX10 cooperates with other transcription factors to activate the expression of key myelin genes in Schwann cells and is therefore a context-dependent, pro-myelination transcription factor. As such, the identification of genes regulated by SOX10 will provide insight into Schwann cell biology and related diseases. While genome-wide studies have successfully revealed SOX10 target genes, these efforts mainly focused on myelinating stages of Schwann cell development. We propose that less-biased approaches will reveal novel functions of SOX10 outside of myelination.

Results: We developed a stringent, computational-based screen for genome-wide identification of SOX10 response elements. Experimental validation of a pilot set of predicted binding sites in multiple systems revealed that SOX10 directly regulates a previously unreported alternative promoter at SOX6, which encodes a transcription factor that inhibits glial cell differentiation. We further explored the utility of our computational approach by combining it with DNase-seq analysis in cultured Schwann cells and previously published SOX10 ChIP-seq data from rat sciatic nerve. Remarkably, this analysis enriched for genomic segments that map to loci involved in the negative regulation of gliogenesis including SOX5, SOX6, NOTCH1, HMGA2, HES1, MYCN, ID4, and ID2. Functional studies in Schwann cells revealed that: (1) all eight loci are expressed prior to myelination and down-regulated subsequent to myelination; (2) seven of the eight loci harbor validated SOX10 binding sites; and (3) seven of the eight loci are down-regulated upon repressing SOX10 function.
\end{abstract}

Conclusions: Our computational strategy revealed a putative novel function for SOX10 in Schwann cells, which suggests a model where SOX10 activates the expression of genes that inhibit myelination during non-myelinating stages of Schwann cell development. Importantly, the computational and functional datasets we present here will be valuable for the study of transcriptional regulation, SOX protein function, and glial cell biology.

Keywords: Comparative sequence analysis, Transcriptional regulation, Ultra-conserved sequences, Myelination, Schwann cells, SOX10

\footnotetext{
* Correspondence: antonell@umich.edu

${ }^{\dagger}$ Equal contributors

${ }^{1}$ Cellular and Molecular Biology Program, University of Michigan Medical

School, Ann Arbor, Ml 48109, USA

${ }^{2}$ Department of Human Genetics, University of Michigan Medical School,

Ann Arbor, Ml 48109, USA

Full list of author information is available at the end of the article
}

(c) The Author(s). 2016 Open Access This article is distributed under the terms of the Creative Commons Attribution 4.0 International License (http://creativecommons.org/licenses/by/4.0/), which permits unrestricted use, distribution, and reproduction in any medium, provided you give appropriate credit to the original author(s) and the source, provide a link to the Creative Commons license, and indicate if changes were made. The Creative Commons Public Domain Dedication waiver (http://creativecommons.org/publicdomain/zero/1.0/) applies to the data made available in this article, unless otherwise stated. 


\section{Background}

Schwann cells produce the myelin sheath in the peripheral nervous system (PNS), which allows rapid saltatory conduction and long-range communication between the central nervous system and innervated muscles and sensory organs. Schwann cell development is directed by a transcriptional hierarchy that promotes the expression of proteins important for migration along peripheral nerves, radial sorting of axons, and the initiation of myelination [1, 2]. Atop this hierarchy sits the transcription factor SOX10, which is critical for the development and long-term function of Schwann cells [3] and is expressed during all stages of Schwann cell development [3, 4].

Three major lines of evidence underscore the importance of SOX10 for the function of Schwann cells. First, ablation of Sox10 expression in mouse models causes: $(i)$ a lack of Schwann cells when performed during early development [4]; (ii) lethality due to peripheral neuropathy when performed in immature Schwann cells [5]; and (iii) demyelination of peripheral nerves when performed in terminally differentiated Schwann cells [6]. Second, dominant-negative SOX10 mutations cause an autosomal dominant disease characterized by peripheral demyelinating neuropathy, central dysmyelinating leukodystrophy, Waardenburg-Shah syndrome, and Hirschsprung disease [7, 8]; the non-PNS phenotypes reflect the role of SOX10 in other neural crest derivatives (i.e., melanocytes and enteric neurons) and in oligodendrocytes. Finally, mutations in SOX10 target genes including those encoding peripheral myelin protein 22 (PMP22), myelin protein zero (MPZ), and gap junction beta 1 (GJB1) cause demyelinating peripheral neuropathy [9-13].

The identification of additional SOX10 response elements and target loci will provide important information on the process of myelination in the peripheral nerve as well as novel target sequences to scrutinize for mutations and modifiers of peripheral neuropathy. Indeed, genome-wide analyses have been essential for characterizing SOX10 biology in Schwann cells [14, 15]; however, these efforts have primarily focused on identifying positive regulators of myelination by examining tissues or cells in a myelinating state. Thus, less-biased approaches are needed to complement the above studies and to identify functions of SOX10 outside of the regulation of promyelinating loci.

Here, we describe a stringent computational strategy to rapidly predict SOX10 response elements in the human genome. Combined with molecular functional studies, this strategy revealed that SOX10 positively regulates the expression of SOX5, SOX6, NOTCH1, HMGA2, HES1, MYCN, ID4, and ID2. Interestingly, each of these genes has a known or predicted role in the negative regulation of glial cell differentiation. As such, we identified a putative novel role for SOX10 in
Schwann cells and present a model where SOX10 activates the expression of negative regulators of myelination to temper the pro-myelinating program during non-myelinating stages of Schwann cell development.

\section{Results}

Genome-wide prediction of SOX10-responsive transcriptional regulatory elements

SOX10 binds to a well-defined consensus sequence ('ACACA' or 'ACAAD'; where 'D' is a G, T, or A nucleotide) as a monomer or as a dimer when two consensus sequences are oriented in a head-to-head fashion $[14,16]$. To identify putative SOX10 binding sites in the human genome, we wrote a Perl script to scan each human chromosome and report all occurrences of the above SOX10 consensus sequence. This revealed over 33 million monomeric consensus sequences (Additional file 1) and $\sim 549,000$ dimeric consensus sequences with an intervening sequence of five to 10 base pairs (Additional file 2).

Multiple-species conservation analysis is an effective approach for predicting non-coding DNA sequences with a role in transcriptional regulation [17]. Importantly, functionally validated SOX10 binding sites have been identified in non-coding genomic sequences that are conserved between human and chicken [18-20]. To prioritize the large dataset of SOX10 consensus sequences, we aligned the human, mouse, and chicken genomes and identified all genomic sequences that are five base pairs or longer (the length of the monomeric SOX10 consensus sequence) and that are identical between these three species. This revealed over two million conserved coding and non-coding genomic segments (Additional file 3).

To develop a panel of prioritized SOX10 consensus sequences for functional studies, we used the rationale that: (1) focusing on conserved dimeric SOX10 consensus sequences will enrich for bona fide SOX10 binding sites; (2) focusing on non-coding sequences will deprioritize sequences that are conserved due to the function of the gene product; and (3) focusing on proximal promoter and intronic sequences will provide a candidate target gene for further studies. Thus, we compared the above datasets to identify dimeric SOX10 consensus sequences that are conserved between human, mouse, and chicken (including the intervening sequence), reside in non-coding sequences, and map to an intron or $2.5 \mathrm{~kb}$ upstream or downstream of a known (RefSeq) human gene. This revealed 238 genomic sequences at 160 loci for further study (Additional file 4). To determine the efficacy of our approach, we further prioritized the above 238 genomic segments by identifying the subset that map to loci with a known or predicted role in myelination (see methods for details). This revealed 57 genomic sequences at 32 loci with a conserved, dimeric SOX10 consensus sequence that resides within an intron or 
directly upstream of a myelin-related transcriptional unit; we named these elements SOX10 Conserved Consensus Sequences (SOX10-CCS; Additional file 5).

\section{Seven conserved SOX10 consensus sequences display regulatory activity in Schwann cells}

Using our computational pipeline, we identified 57 regions that harbor conserved head-to-head SOX10 consensus sequences at loci with a known or predicted role in myelination. To test if these sequences are active in Schwann cells in vitro, a region surrounding each consensus sequence (Additional file 5) was amplified from human genomic DNA and cloned upstream of a minimal promoter directing the expression of a luciferase reporter gene. The regulatory activity of each genomic segment was tested in cultured rat Schwann (S16) cells [21, 22], which express endogenous SOX10 [19]. The luciferase expression directed by each genomic segment was determined in luciferase activity assays compared to a control vector with no genomic insert ('Empty'). Seven of the 57 genomic segments demonstrated a greater than 2.5-fold increase in luciferase activity compared to the empty vector in S16 cells (Fig. 1): SOX10-CCS-01 (3.7-fold increase; maps to $P A X 7$ ), SOX10-CCS-13 (54-fold increase; maps to SOX6), SOX10-CCS-18 (82-fold increase; maps to SOX5), SOX10-CCS-19 (49-fold increase; maps to SOX5), SOX10-CCS-39 (5.9-fold increase; maps to TCF7L2), SOX10-CCS-43 (25-fold increase; maps to BCAS3), and SOX10-CCS-51 (2.6-fold increase; maps to NFIB). These data suggest that these seven genomic sequences (Table 1) are potential SOX10 response elements.

\section{The SOX10 consensus sequence is required for the orientation-independent activity of three regulatory elements at SOX5, SOX6, and NFIB}

To determine if the regulatory activity of the seven genomic segments is dependent on the orientation of the DNA sequence, we retested the activity of each segment in both the 'forward' and 'reverse' orientation relative to a construct with no genomic insert ('Empty') within our reporter gene construct in S16 cells. This revealed three genomic segments that enact a greater than 2.5-fold increase in luciferase activity in both orientations (Fig. 2a): SOX10-CCS-13 (72-fold forward and 9-fold reverse), SOX10-CCS-19 (70-fold forward and 33-fold reverse), and SOX10-CCS-51 (4-fold forward and 9-fold reverse). To assess the specificity of these results to Schwann cells, we tested each of the seven genomic segments in both orientations in cultured mouse motor neurons (MN1 cells) [23], which do not express endogenous SOX10 [19]. None of the genomic segments enact a greater than 2.5-fold increase in luciferase activity in both orientations in MN1 cells suggesting that our data in S16 cells is Schwann-cell specific; however, three had low levels of activity in only the forward orientation in MN1 cells (Additional file 6: Figure S1): SOX10-CCS-39 (5.5-fold), SOX10-CCS-43 (6.7-fold), and SOX10-CCS-51 (4-fold).

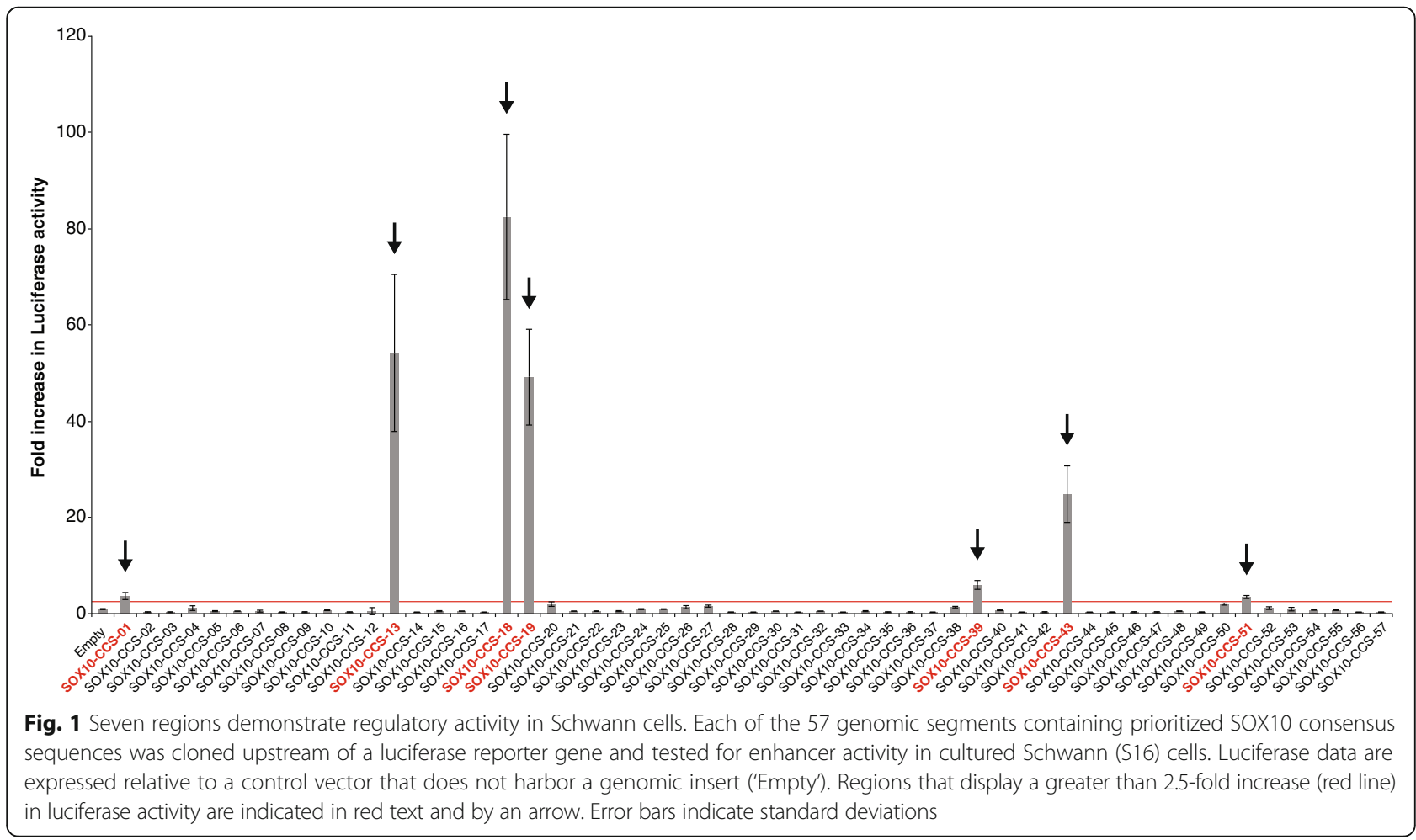


Table 1 Seven genomic segments with regulatory activity in Schwann cells

\begin{tabular}{llll}
\hline Element ID & Locus & UCSC coordinates $^{a}$ & SOX10 Consensus sequence $^{\text {b }}$ \\
\hline SOX10-CCS-01 & PAX7 & chr1:18,854,300-18,855,055 & $\underline{\text { ACAAACTCATTAAACTTGT }}$ \\
SOX10-CCS-13 & SOX6 & chr11:16,334,301-16,335,278 & $\underline{\text { ACAATCAAGCATTGT }}$ \\
SOX10-CCS-18 & SOX5 & chr12:24,058,988-24,059,872 & $\underline{\text { ACAAAAATGTATTGT }}$ \\
SOX10-CCS-19 & SOX5 & chr12:24,059,397-24,060,164 & $\underline{\text { ACACAGAACATTATTGT }}$ \\
SOX10-CCS-39 & TCF7L2 & chr10:114,894,980-114,895,808 & $\underline{\text { ACAATCCCCAAGATTTTGT }}$ \\
SOX10-CCS-43 & BCAS3 & chr17:56,683,905-56,684,657 & $\underline{\text { ACACATTAATAACGTTTTGT }}$ \\
SOX10-CCS-51 & NFIB & chr9:14,299,332-14,299,796 & $\underline{\text { ACAATCTGTTCTITGT }}$ \\
\hline
\end{tabular}

${ }^{a}$ Coordinates refer to the March 2006 UCSC Genome Browser Human assembly (hg18)

${ }^{b}$ SOX10 consensus sequences are indicated in bold, underlined text

To test the necessity of the conserved SOX10 consensus sequence for the observed activity associated with the seven genomic segments described above, we deleted the dimeric SOX10 site along with the intervening sequence in each construct ( $\triangle \mathrm{SOX} 10)$ and compared the activity to the wild-type genomic segment using the more active orientation. This revealed three genomic segments that display at least a $50 \%$ reduction in activity upon deleting the SOX10 consensus sequence (Fig. 2b): SOX10-CCS-13, SOX10-CCS-19, and SOX10CCS-51. Combined, our data are consistent with these three genomic segments-at the SOX6, SOX5, and NFIB loci, respectively-representing Schwann cell enhancers that harbor functional SOX10 binding sites.

\section{SOX10 is required for the activity of the three regulatory elements at SOX5, SOX6, and NFIB}

To test if SOX10 induces the activity of SOX10-CCS-13, SOX10-CCS-19, and SOX10-CCS-51, we co-transfected each reporter gene construct with or without a construct to express wild-type SOX10 in MN1 cells, which do not express endogenous SOX10 [8, 19]. Subsequently, we compared the activity of each construct in the presence or absence of SOX10 expression. There was a 1,000fold increase in the activity of SOX10-CCS-13 and a 200-fold increase in the activities of SOX10-CCS-19 and SOX10-CCS-51 in the presence of SOX10 (Fig. 3a).

SOX8, SOX9, and SOX10 belong to the SOXE family of transcription factors, which bind to nearly identical sequence motifs [24]. To test if SOX10 specifically regulates SOX10-CCS-13, SOX10-CCS-19, and SOX10-CCS-51, we co-transfected each reporter construct with or without a construct to express SOX8 or SOX9 in MN1 cells $[19,25]$ and compared the effect on regulatory activity with that induced by SOX10. In the presence of SOX8 we observed a 140-fold, $\sim 75$-fold, and $\sim 50$ fold increase in the activity of SOX10-CCS-13, SOX10-CCS-19, and SOX10-CCS-51, respectively (Additional file 7: Figure S2). In the presence of SOX9 we observed a $~ 350$-fold, 150-fold, and 80-fold increase in the activity of SOX10-CCS-13, SOX10-CCS-19, and SOX10-CCS-51, respectively (Additional file 7:
Figure S2). Importantly, SOX8 and SOX9 did not increase the luciferase activity of these regions to the same level as SOX10 (Additional file 8: Figure S3), suggesting that SOX10 has a higher affinity for the sequences within SOX10-CCS-13, SOX10-CCS-19, and SOX10-CCS-51.

SOX10 is known to synergistically interact with other transcription factors to enact gene expression [OCT6, BRN2, and EGR2 in Schwann cells, OLIG2 and MYRF in oligodendrocytes [26], and PAX3 and MITF in melanocytes [27]]. Thus, we wanted to determine if the most well-characterized co-factor in Schwann cells (EGR2) works synergistically to activate these elements. EGR2, a master regulator of Schwann cell myelination, is regulated by SOX10, OCT6, and BRN2 [28]. SOX10 and EGR2 synergistically regulate key myelin genes such as PMP22 [10], MPZ [11], and GJB1 [9]. We co-transfected SOX10-CCS-13, SOX10-CCS-19, and SOX10-CCS-51 reporter constructs with a construct to express EGR2 and SOX10 in MN1 cells and compared the effect on regulatory activity with that induced by SOX10 alone (Additional file 8: Figure S3). In the presence of EGR2 we observed a moderate increase in luciferase activity of SOX10-CCS-13 ( 2.2-fold), SOX10-CCS-19 ( 12-fold) and SOX10-CCS-51 ( 10-fold) (Additional file 8: Figure S3). However, in the presence of both EGR2 and SOX10 we did not see an increase in activity above that induced by SOX10 alone (even though an equivalent amount of SOX10 expression vector was transfected in each experiment). These data suggest that the three regions are primarily regulated by SOX10 and that EGR2 and SOX10 do not act synergistically upon them.

To determine if SOX10 is necessary for the activity of SOX10-CCS-13, SOX10-CCS-19, and SOX10-CCS-51 in Schwann cells, S16 cells were transfected with each SOX10-CCS luciferase reporter gene construct along with a construct to express a dominant-negative mutant form of SOX10 (E189X), which interferes with the function of endogenous SOX10 [8]. Importantly, E189X SOX10 has been shown to specifically reduce the activity of genomic segments harboring SOX10 binding sites in luciferase assays [29]. We observed a greater than $85 \%$ 


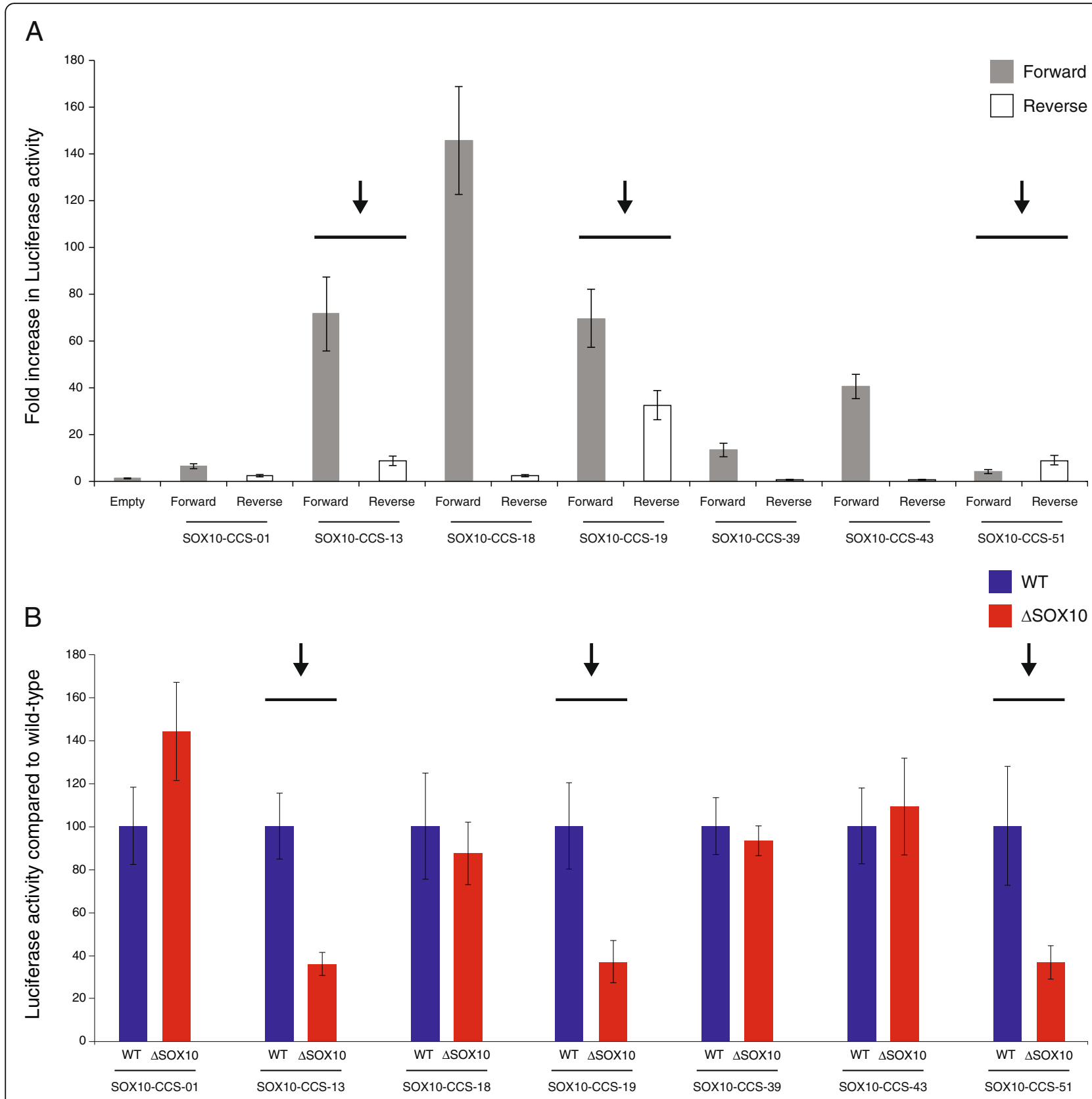

Fig. 2 Three genomic segments require the SOX10 consensus sequence for activity in Schwann cells. a The seven active regions from Fig. 1 were tested in forward and reverse orientation in rat Schwann (S16) cells. Luciferase data are expressed relative to a control vector without a genomic segment ('Empty'). Error bars indicate standard deviations and arrows and lines indicate genomic segments that are active in both orientations. $\mathbf{b}$ Luciferase reporter gene constructs containing either the wild-type sequence (WT) or the sequence lacking the SOX10 consensus sequence(s) ( $\Delta$ SOX10) were transfected into S16 cells and tested in luciferase assays. The luciferase activity associated with each $\Delta$ SOX10 construct is expressed relative to the respective wild-type construct. Error bars indicate standard deviations and arrows and lines indicate genomic segments with a required SOX10 consensus sequence

reduction in the activity of all three genomic segments upon co-transfection with E189X SOX10 (Fig. 3b). Combined, our data indicate that SOX10 is required for the in vitro enhancer activity of SOX10-CCS-13, SOX10-CCS-19, and SOX10-CCS-51.
SOX10-CCS-13 is a previously unreported, alternative Sox6 promoter

Examination of SOX10-CCS-13 on the UCSC Genome Browser revealed that the SOX10 consensus sequence within this genomic segment is also conserved between 


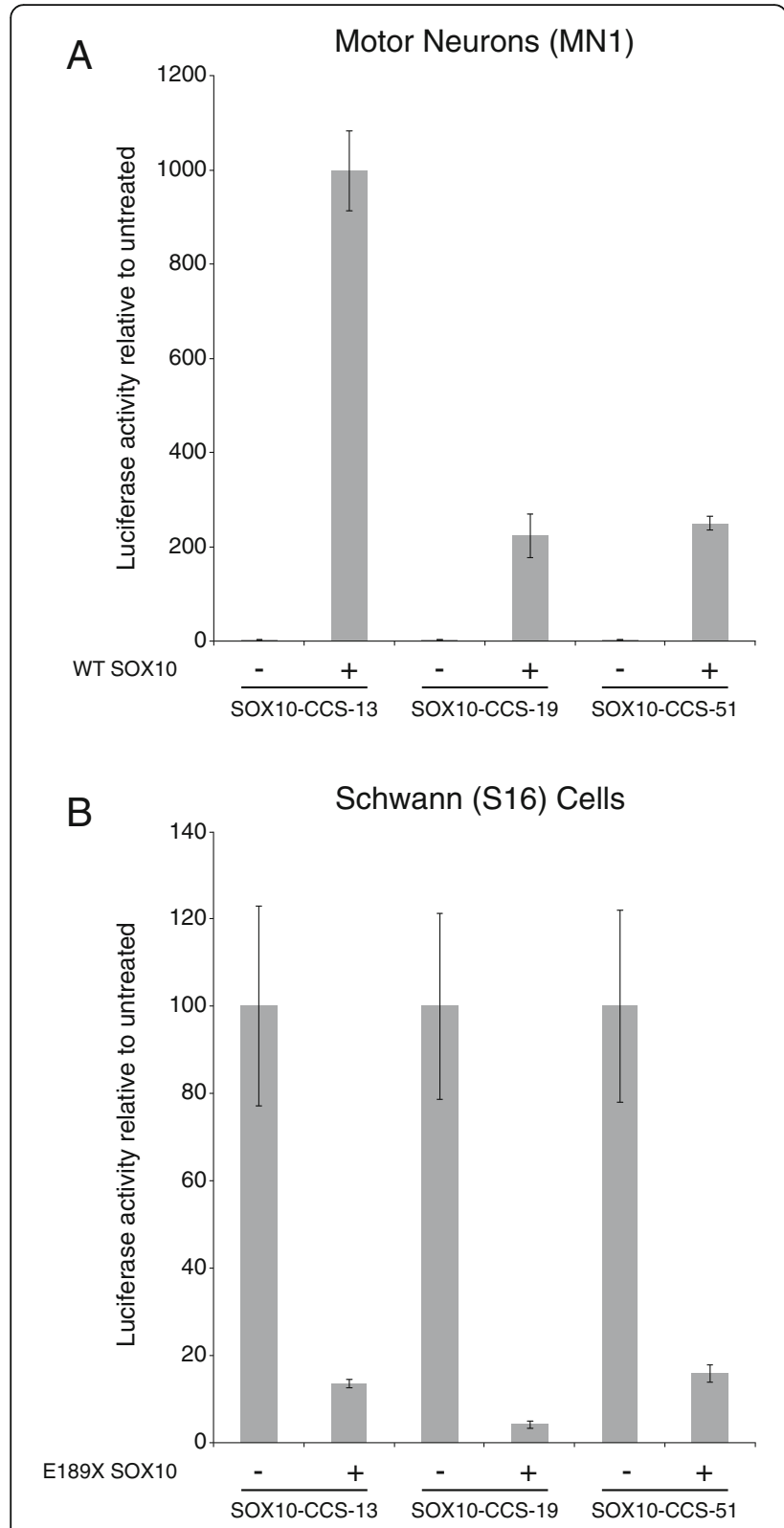

Fig. 3 SOX10 is required for the regulatory activities of SOX10-CCS-13, SOX10-CCS-19 and SOX10-CCS-51. a Luciferase reporter gene constructs harboring SOX10-CCS-13, SOX10-CCS-19 or SOX10-CCS-51 were transfected into mouse motor neurons (MN1) with or without a construct to express wild-type SOX10. The luciferase activity associated with each construct in the presence of SOX10 is expressed relative to that of the construct in the absence of SOX10. $\mathbf{b}$ Luciferase reporter gene constructs harboring SOX10-CCS-13, SOX10-CCS-19 or SOX10CCS-51 were transfected into rat Schwann (S16) cells with or without a construct to express dominant-negative (E189X) SOX10. The luciferase activity associated with each construct in the presence of E189X SOX10 is expressed relative to that of the construct in the absence of E189X SOX10. Error bars indicate standard deviations in both panels

human and zebrafish (data not shown) further suggesting an important role for this SOX10 response element in jawed vertebrates. Therefore, to validate our computational approach we pursued additional analyses of SOX10-CCS13, which resides at the SOX6 locus. Closer scrutiny of the SOX6 locus on the UCSC Genome Browser [30] revealed seven unique SOX6 mRNA isoforms in human, mouse, or rat, distinguished by alternative, non-coding first exons. Interestingly, SOX10-CCS-13 maps directly upstream of the 3'-most alternative first exon, which we named SOX6 exon $1 \mathrm{G}$ (Fig. 4). We therefore hypothesized that SOX10CCS-13 acts as an alternative promoter at SOX6. To test this, we performed 5'-rapid amplification of cDNA ends (5'-RACE). Briefly, a cDNA library was generated using RNA isolated from cultured rat Schwann (S16) cells and a reverse primer in exon 5 of the rat Sox 6 gene. Subsequently, nested PCR was performed using reverse primers in exon 4 and then exon 3 of Sox6. The PCR products were cloned, sequenced, and aligned to the rat Sox6 locus. These analyses revealed the presence of five unique Sox6 transcription start sites in cultured Schwann cells with 14 of the 44 Sox6-specific sequences mapping directly downstream of SOX10-CCS-13 (Fig. 4). Analysis of RNA-seq data generated in S16 cells (Antonellis and Law, in preparation) also revealed reads that map to Sox6 exon $1 G$ (Fig. 4), with split reads into downstream exons, but no split reads into upstream exons (data not shown). Additionally, we were able to amplify and sequenceverify a full length Sox6 mRNA that originates at exon $1 \mathrm{G}$ in S16 cells (Fig. 4).

To assess the in vivo relevance of SOX10-CCS-13 we analyzed recently published SOX10 ChIP-seq data performed on nuclei isolated from rat spinal cord and sciatic nerve $[14,15]$. Furthermore, to establish that this genomic segment resides in open chromatin we performed DNaseseq analysis on nuclei isolated from cultured rat Schwann (S16) cells (see below). The SOX10 ChIP-seq analyses revealed that SOX10 binds to SOX10-CCS-13 in relevant tissues in vivo and the DNase-seq experiment revealed that this genomic segment resides in open chromatin in cultured Schwann cells (Fig. 4, green box). Combined, these data support our conclusion that SOX10-CCS-13 is a SOX10 response element in Schwann cells. Interestingly, these genome-wide functional studies revealed additional SOX10 binding sites at Sox6 in vivo further supporting the notion that Sox6 is a SOX10 target gene (e.g., brown highlighted region in Fig. 4). Combined, our data indicate that SOX10-CCS-13 represents an internal, alternative, SOX10-responsive promoter at Sox6.

\section{SOX10 is necessary and sufficient for the expression of Sox6 transcripts harboring exon $1 \mathrm{G}$}

To determine if SOX10 is sufficient to direct the expression of Sox6 transcripts, we performed RT-PCR using primers designed in Sox6 exon $1 G$ and exon 2 in regions conserved between rat and mouse. While these primers amplify Sox6 transcripts containing exon 1G from a 


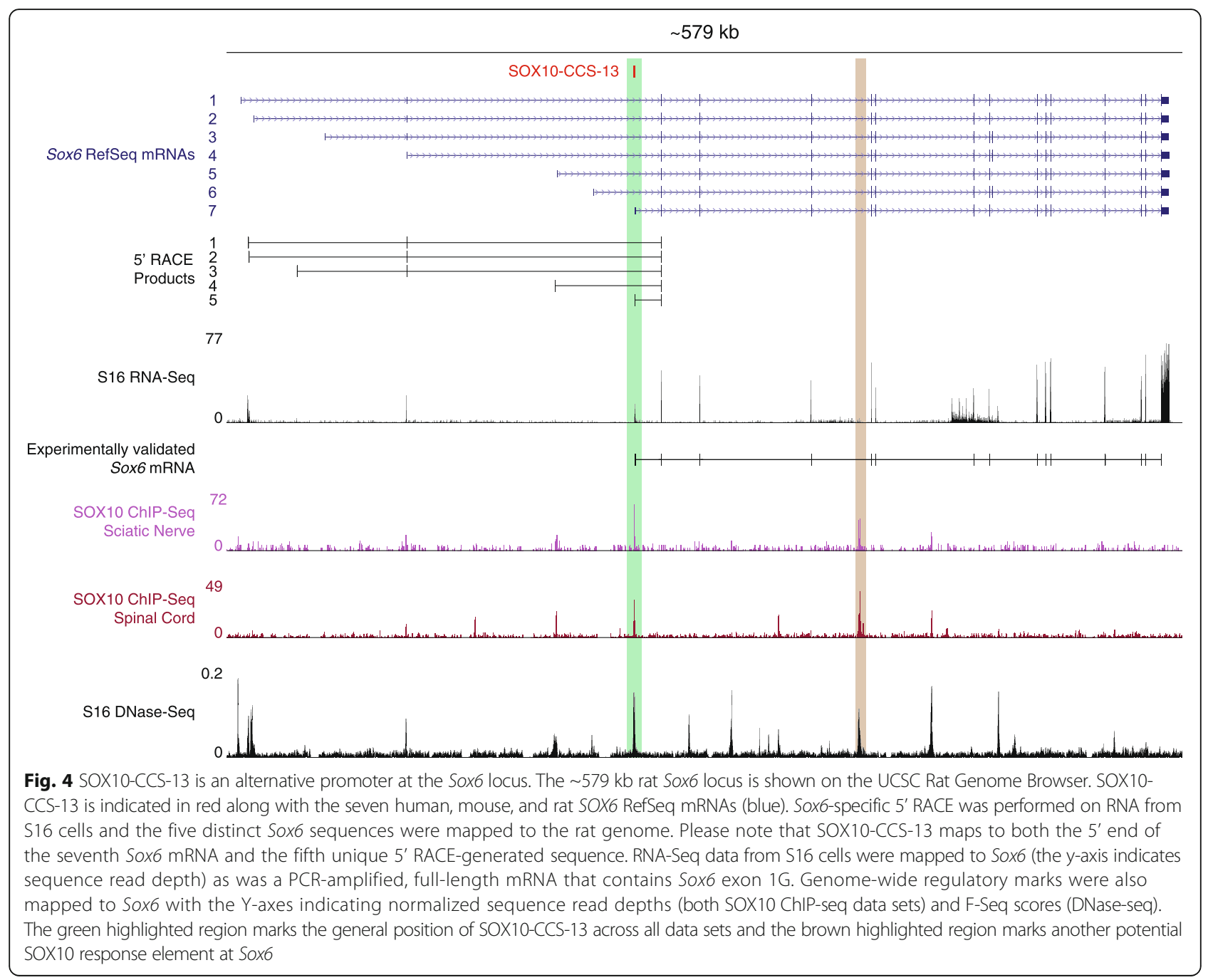

cDNA library generated from S16 RNA, we were not able to amplify these transcripts from a cDNA library generated from cultured mouse motor neurons (MN1 cells), which do not express endogenous SOX10 (Fig. 5a). However, when MN1 cells were transfected with a construct to express wild-type SOX10, Sox6 transcripts containing exon $1 \mathrm{G}$ were detected and verified by DNA sequence analysis. Mock transfection or transfection with a construct to express a non-functional mutant version of SOX10 (E189X) [8] did not allow amplification of Sox6 transcripts containing exon 1G (Fig. 5a). Thus, SOX10 is sufficient to activate the expression of Sox6 transcripts harboring exon 1G in MN1 cells.

To determine if SOX10 is necessary for the expression of Sox6 transcripts containing exon 1G in Schwann cells, we treated S16 cells with a previously validated siRNA against Sox10 $[15,20]$ and tested for an effect on total Sox6 mRNA levels and for an effect on the level of transcripts containing exon 1G. This analysis revealed a $\sim 70 \%$ decrease in both total Sox6 expression and in the expression of transcripts containing exon 1G (Fig. 5b), consistent with SOX10 regulating the promoter activity of SOX10-CCS-13 in Schwann cells. Combined, our data indicate that SOX10 is both necessary and sufficient for the expression of Sox6 mRNA isoform 7 (Fig. 4) in our in vitro cell culture model systems.

\section{SOX10 regulates the expression of genes that inhibit myelination}

Our stringent computational and functional analyses rapidly identified a previously unreported SOX10-responsive promoter at the SOX6 locus. Importantly, this finding was facilitated by the knowledge of a well-defined SOX10 consensus sequence and reports that SOX10 binding sites can be conserved among vertebrate species including human and chicken [18-20]. To determine if our conservation analysis combined with whole genome datasets can reveal a set of high-confidence SOX10 response elements for further study we: (1) utilized available SOX10 ChIP-seq data generated from rat Schwann cell nuclei in vivo [14]; 


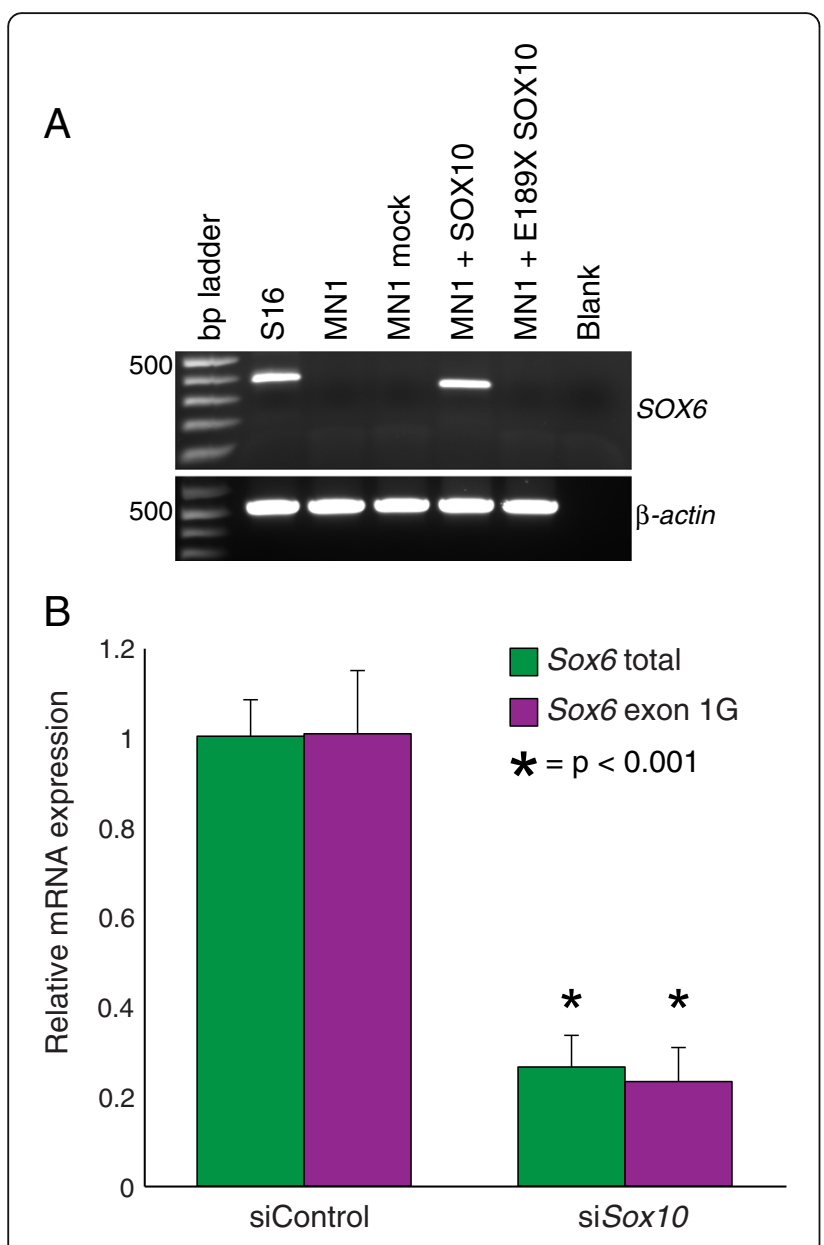

Fig. 5 SOX10 is necessary and sufficient for SOX6 expression. a RT-PCR was performed to detect the expression of Sox6 transcripts harboring exon $1 G$ using CDNA isolated from S16 cells, MN1 cells, or MN1 cells transfected with no expression construct (mock) or a construct to express wild-type or dominant-negative (E189X) SOX10. Base pair (bp) ladders are indicated on the left. RT-PCR for $\beta$-actin and samples including no cDNA ('Blank') were employed as positive and negative controls, respectively. Please note that while the same primers were used for each reaction, the rat (S16) PCR product was 402 base pairs and the mouse (MN1) PCR product was $349 \mathrm{bp}$; the rat genome harbors a 53 base pair rat-specific insertion, which we confirmed via DNA sequence analysis. b Rat Schwann (S16) cells were treated with a control siRNA (left side) or a siRNA targeted against Sox10 (right side). Quantitative RT-PCR was used to measure expression levels of total Sox6 (green bars) or Sox6 exon 1G-containing (purple bars) transcripts. Error bars indicate standard deviations

(2) performed DNase-seq on cultured rat Schwann (S16) cell nuclei; and (3) identified 67,482 non-coding SOX10 monomeric consensus sequences conserved between human, mouse, and chicken (data not shown), and converted them to the rat genome $[\mathrm{rn} 5 ; 61,133$ (90.5 \%) were successfully converted]. Intersecting these three data sets revealed 214 rat genomic segments that harbor conserved SOX10 consensus sequences and that map to SOX10 ChIP-seq and DNase-seq peaks (Additional file 9; these genomic segments were computationally extracted as SOX10 ChIP-seq peaks). To determine if this approach identified specific biological pathways, we extracted the name of the rat RefSeq gene closest to each region-the 214 genomic segments map to 191 known genes (Additional file 9)-and performed a gene ontology search using the overrepresentation test for biological processes (geneontology.org). This analysis revealed 183 biological processes with a $p$-value less than 0.05 and 37 biological processes that showed a greater than five-fold enrichment compared to the human genome. Ten of the identified biological processes directly relate to myelinating glia, which all resided in the top 14 enriched terms (Table 2). Therefore, this combined strategy provided a highly confident set of 214 SOX10-response elements at 191 loci for future functional studies aimed at better understanding the biological process of myelination (Additional file 9).

Interestingly, three of the 10 gene ontology biological processes that relate to myelination specifically relate to negative regulation of gliogenesis, which was due to the presence of six genes: NOTCH1, HMGA2, HES1, MYCN, ID4, and ID2 (Table 2). Computational analyses revealed eight SOX10 consensus sequences within DNase-seq and SOX10 ChIP-seq peaks at these six loci (Table 3). To determine if NOTCH1, HMGA2, HES1, MYCN, ID4, and ID2 harbor bona fide SOX10 response elements, we amplified genomic regions surrounding the SOX10 consensus sequences using rat genomic DNA and cloned each genomic segment (in both the 'forward' and 'reverse' orientation) upstream of a minimal promoter directing luciferase expression. The regulatory activity of each genomic segment was tested in S16 cells as described above. This revealed five genomic segments ('regions' or ' $R$ ') that directed reporter gene activity at least 2.5 -fold higher than the empty control vector in both orientations: Notch1-R1 (4.7-fold forward and 56-fold reverse), Hmga2-R2 (93.7fold forward and 87-fold reverse), Hes1-R1 (22-fold forward and 7.6-fold reverse), Mycn-R1 (28-fold forward and 16-fold reverse) and $I d 2-R 1$ (8.9-fold forward and 4.1-fold reverse) (Fig. 6a). Regions Notch1-R2 (7.6-fold) and Id4-R1 (8.6-fold) directed reporter gene activity at least 2.5-fold higher than the empty control vector only in the forward orientation (Fig. 6a). Hmga2-R1 was not active in either orientation and was excluded from further analysis. Thus, we identified seven genomic sequences at six loci (NOTCH1, HMGA2, HES1, MYCN, ID4, and ID2) that display regulatory activity in Schwann cells.

To determine if the identified SOX10 consensus sequences (Table 3) are important for the regulatory activity of the seven active regions described above (Fig. 6a) we deleted the SOX10 consensus sequence from each construct (termed ' $\triangle \mathrm{SOX} 10$ ' in Fig. $6 \mathrm{~b}$ ) and compared the activity to the wild-type construct using the more 
Table 2 Gene ontology annotations of loci harboring conserved SOX10 binding sites

\begin{tabular}{|c|c|c|c|c|c|}
\hline GO biological process complete & Human & Our list & Expected & $P$-value & Loci \\
\hline Negative regulation of oligodendrocyte differentiation & 12 & 4 & 0.1 & $2.74 \mathrm{E}-02$ & HES1, ID2, NOTCH1, ID4 \\
\hline Negative regulation of glial cell differentiation & 25 & 6 & 0.21 & $6.34 \mathrm{E}-04$ & HES1, ID2, NOTCH1, HMGA2, MYCN, ID4 \\
\hline Regulation of astrocyte differentiation & 25 & 6 & 0.21 & $6.34 \mathrm{E}-04$ & HES1, ID2, NOTCH1, HMGA2, MYCN, ID4 \\
\hline Regulation of oligodendrocyte differentiation & 28 & 5 & 0.23 & $3.29 \mathrm{E}-02$ & HES1, TCF7L2, ID2, NOTCH1, ID4 \\
\hline Negative regulation of gliogenesis & 34 & 6 & 0.28 & $3.77 \mathrm{E}-03$ & HES1, ID2, NOTCH1, HMGA2, MYCN, ID4 \\
\hline Oligodendrocyte differentiation & 60 & 10 & 0.5 & $9.43 \mathrm{E}-07$ & $\begin{array}{l}\text { SOX6, NTRK2, PTPRZ1, SOX8, SOX10, TCF7L2, ID2, } \\
\text { NOTCH1, SOX5, ID4 }\end{array}$ \\
\hline Regulation of gliogenesis & 74 & 10 & 0.61 & $6.96 \mathrm{E}-06$ & $\begin{array}{l}\text { PTPRZ1, SOX8, HES1, SOX10, TCF7L2, ID2, NOTCH1, } \\
\text { HMGA2, MYCN, ID4 }\end{array}$ \\
\hline Regulation of glial cell differentiation & 54 & 7 & 0.45 & $3.23 \mathrm{E}-03$ & HES1, TCF7L2, ID2, NOTCH1, HMGA2, MYCN, ID4 \\
\hline Glial cell differentiation & 135 & 13 & 1.12 & $1.22 \mathrm{E}-06$ & $\begin{array}{l}\text { SOX6, PTPRZ1, NTRK2, SOX8, HES1, SOX10, TCF7L2, } \\
\text { ID2, NOTCH1, PPAP2B, SOX5, ID4, PARD3 }\end{array}$ \\
\hline Gliogenesis & 168 & 13 & 1.39 & 1.66E-05 & $\begin{array}{l}\text { SOX6, PTPRZ1, NTRK2, SOX8, HES1, SOX10, TCF7L2, } \\
\text { ID2, NOTCH1, PPAP2B, SOX5, ID4, PARD3 }\end{array}$ \\
\hline
\end{tabular}

active orientation. Notch1-R1, Notch1-R2, Hmga2-R2, Hes1-R1, and Id2-R1 contain dimeric SOX10 consensus sequences, which were deleted along with the intervening sequence. $M y c n-R 1$ contains a monomeric consensus sequence $(\triangle \mathrm{SOX} 10-1)$ and a dimeric consensus sequence ( $\triangle \mathrm{SOX} 10-2)$, which were independently deleted. Id4-R1 contains a dimeric consensus sequence with a 20 basepair intervening sequence. Since this intervening sequence is longer than those previously observed for validated dimeric SOX10 binding sites [16, 18-20, 29, 31] we studied each monomer independently. Specifically, we deleted the dimeric consensus sequence along with intervening sequence $(\triangle \mathrm{SOX} 10-1)$, the first monomer only $(\triangle \mathrm{SOX} 10-$ $2)$, and the second monomer only ( $\triangle$ SOX $10-3)$. Deleting the SOX10 consensus sequences in regions Notch1-R1,

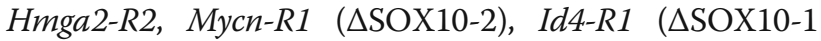
and $\triangle S O X 10-3)$, and $I d 2-R 1$ reduced luciferase activity in S16 cells by at least $50 \%$ (Fig. 6b), indicating that the SOX10 consensus sequences in these five regions are important for their regulatory activity. In contrast, deleting the SOX10 consensus sequences in Notch1-R2 and Hes1-
$R 1$ did not reduce the enhancer activity associated with these genomic segments (Fig. 6b).

We have shown that SOX10 directly regulates SOX6 in Schwann cells (see above). To determine if SOX10 positively regulates the expression of Notch1, Hmga2, Hes1, Mycn, Id4, Id2, and Sox5 in cultured rat Schwann (S16) cells we again utilized the Sox10 siRNA that has been shown to efficiently down-regulate Sox10 expression $[15,20]$. After isolation of mRNA at $24 \mathrm{~h}$ posttransfection, qRT-PCR shows that Sox10 depletion in S16 cells results in the reduced expression of all of the above genes except for Hmga2 (Fig. 6c). Similar findings-including the absence of reduced Hmga2 expression-were observed upon repressing SOX10 function in vivo using primary Schwann cells; however, this system is prone to variability due to heterogeneous cell populations (Additional file 10: Figure S4).

To directly test if NOTCH1, HMGA2, HES1, MYCN, ID4, ID2, SOX5, and SOX6 are developmentally regulated during myelination in vivo we examined mRNA levels at three timepoints in rat sciatic nerve $(n=3$ at

Table 3 Eight genomic segments within loci that inhibit glial cell differentiation

\begin{tabular}{|c|c|c|}
\hline Element ID & UCSC Coordinates $^{a}$ & SOX10 Consensus Sequence ${ }^{b}$ \\
\hline Notch1-R1 & chr3:9,307,836-9,308,296 & $\underline{\text { ACAATGGGGCCTCTGT }}$ \\
\hline Notch1-R2 & chr3:9,308,175-9,309,096 & $\underline{\text { ACAATCGGCTTTGT }}$ \\
\hline Hmga2-R1 & chr7:65,390,088-65,391,287 & CTTAGACACAGCACTT \\
\hline Hmga2-R2 & chr7:65,427,912-65,428,606 & $\underline{\text { ACACAGGCCCCTCTTTGT }}$ \\
\hline Hes1-R1 & 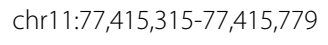 & $\underline{\text { TGTGTGAGCGCCATGTGT }}$ \\
\hline Mycn-R1 & chr6:51,229,947-51,230,533 & $\underline{\text { ACAATGGCCTCTITCTACAGACAAT }}$ \\
\hline $\mid d 4-R 1$ & chr17:18,701,460-18,702,118 & 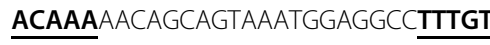 \\
\hline $\mid d 2-R 1$ & chr6:53,090,794-53,091,254 & ACAAGAAACACATTGT \\
\hline
\end{tabular}

${ }^{a}$ Coordinates refer to the March 2012 UCSC Genome Browser Rat assembly (rn5)

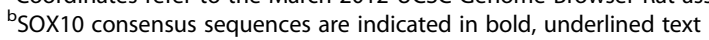




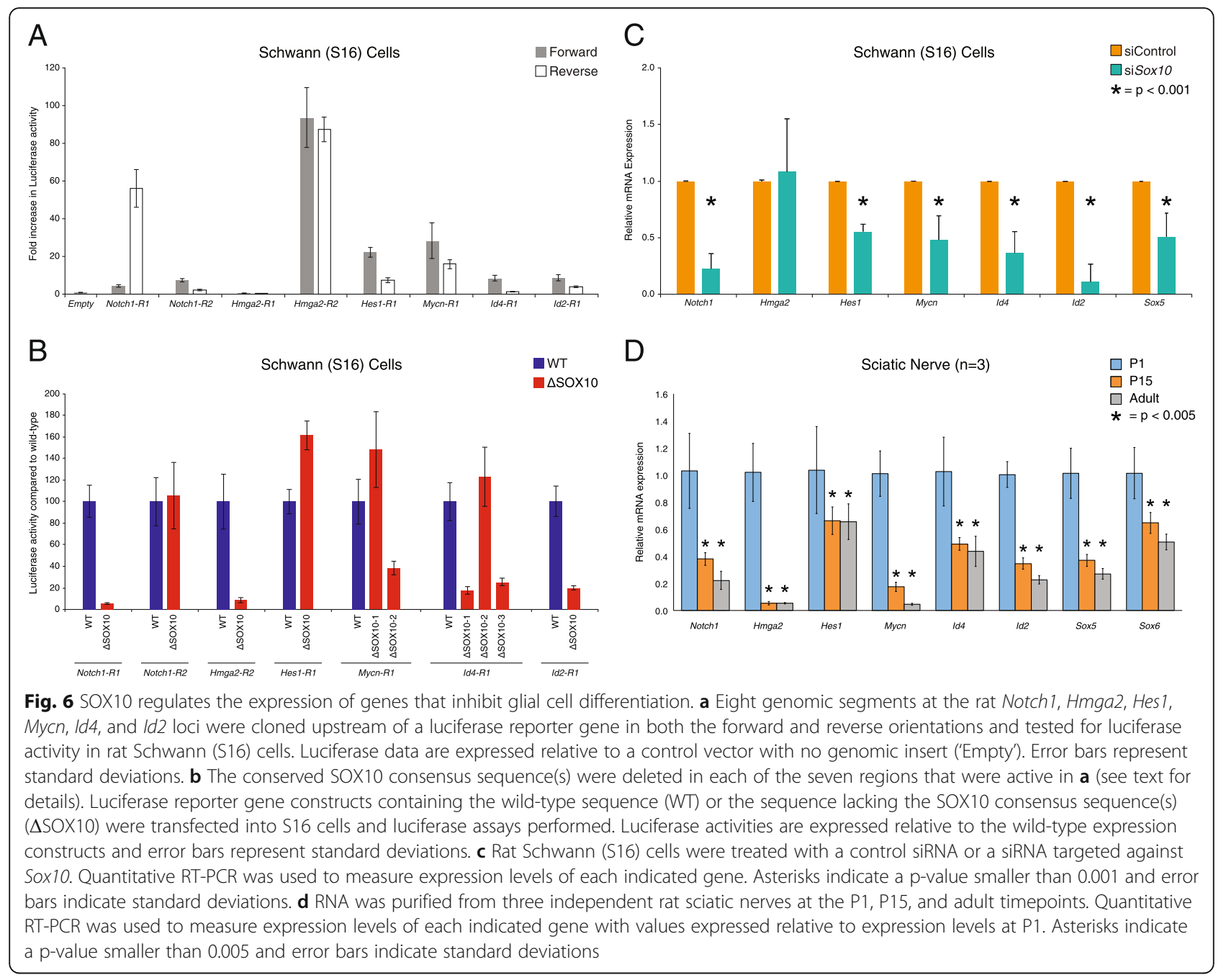

each timepoint). P1 corresponds to the onset of myelination, P15 is a peak timepoint of myelination in the PNS, and adult sciatic nerve is a timepoint where active myelination has subsided. Interestingly, the expression of all seven genes tested (Notch1, Hmga2, Hes1, Mycn, $I d 4, I d 2$, Sox5, and Sox6) are highest at P1 and then repressed at P15 and adult, consistent with a role in repressing precocious myelination (Fig. 6d).

\section{Discussion}

Previous efforts have identified SOX10 binding sites and target genes in Schwann cells [14, 15]. These efforts, and others [32], have utilized a variety of experimental methodologies to identify putative SOX10 regulatory elements across diverse SOX10-positive tissues. While each approach uncovered novel SOX10 response elements, no single method has been successful in the identification of all SOX10 response elements. While the strategies presented here also are unable to fully capture all SOX10 binding sites, the combination of multiple datasets and methodologies generally yields a stronger predictive power for identifying regulatory regions compared to any one individual method [33]. Indeed, when we combined our DNase-seq data with previously generated SOX10 ChIP-seq data [14], we were able to quickly prioritize and validate novel SOX10 response elements near genes with a known role in myelination. While previous efforts to identify SOX10 response elements focused on the required function of SOX10 in cultured cells or tissues at specific developmental stages, our computational approach utilizes sequence conservation to identify putative SOX10 regulatory regions throughout the genome in a tissue-independent manner. The combination of our less-biased (albeit less biologically relevant) computational approach with DNase-seq and ChIP-seq is likely the reason that we were able to identify specific repressors of myelination as putative SOX10 target genes (e.g., these would not have been identified in myelinating Schwann cells). As such, we feel that the datasets generated here will be useful to investigators 
studying comparative genomics, SOX protein function, and Schwann cell biology. First, the conserved sequences we identified could be used to similarly prioritize consensus sequences for other transcription factors important for vertebrate development. Second, the SOX10 consensus sequences we identified could be used to prioritize putative binding sites in other SOX10-positive cells including oligodendrocytes, melanocytes, and developing enteric nervous system neurons [34]. Finally, our DNase-seq data from rat Schwann (S16) cells will be useful for anyone studying transcriptional regulatory elements, highly expressed genes, or any other nuclear structure characterized by open chromatin in myelinating Schwann cells; S16 cells express many myelin-related genes (e.g., $P M P 22, M P Z, M B P$, and $M A G)$ and transcription factors (e.g., SOX10 and EGR2) and are biochemically similar to myelinating Schwann cells [35].

The analyses performed here may also provide insight into the nucleotide requirements for a SOX10 response element. We functionally evaluated a total of 62 conserved, genomic segments that harbor a predicted dimeric SOX10 binding site. Interestingly, simply cloning a highly conserved dimeric SOX10 consensus sequence upstream of a minimal promoter was not enough to enact regulatory activity in cultured Schwann cells as evidenced by the 50 genomic segments with no (or very low) regulatory activity in Fig. 1. While there are many possible explanations for this finding, one is that there are nucleotide-specific requirements for the intervening sequences (i.e., the nucleotides between the head-tohead monomeric sites). To assess this, we compared the length and GC content of all 62 dimeric sites to those dimeric sites that were both active and required for the observed regulatory activity $(n=7)$. While there was no significant difference in the average intervening sequence length between the two groups, the seven active sites all had intervening sequence lengths between five and eight nucleotides consistent with previous reports that six basepairs provides the ideal spacing between monomers [16]. Interestingly, there was a marked difference in the GC content when comparing the total population of intervening sequences (GC content $=35 \%$ ) to the intervening sequences in the active dimeric sites (GC content $=61 \%$ ). These data are consistent with the high GC content of the intervening sequences within previously validated dimeric SOX10 binding sites $[13,18-20,29]$ and with a ' $G$ ' nucleotide being the most commonly observed nucleotide after the core motif [14]. Thus, future predictions of dimeric SOX10 binding sites should allow for high GC content and five to eight basepairs between the head-tohead consensus sequences.

Our efforts predicted eight putative SOX10 target loci with a known role in repressing glial cell differentiation:
NOTCH1, HMGA2, HES1, MYCN, ID2, ID4, SOX5, and SOX6. These findings were unexpected due to the known role of SOX10 in regulating the expression of genes that encode myelin proteins (e.g., $M B P, M P Z$, and PMP22) $[10,12,13,31,36,37]$. We showed that all eight loci are developmentally regulated during myelination in vivo in a manner consistent with a role in inhibiting glial cell differentiation. We were also able to functionally validate a SOX10 binding site at seven of the eight loci. We identified a SOX10 ChIP-seq peak at HES1 and luciferase assays demonstrated that this genomic segment has strong enhancer activity (Fig. 6a). However, deletion of the predicted SOX10 binding sites in Hes1-R1 (Table 3) did not reduce luciferase activity. Further mutagenesis of this genomic segment will be required to identify sequences necessary for the observed activity, which may reveal a degenerate SOX10 consensus sequence. When we depleted SOX10 activity in Schwann cells in vitro and in vivo seven of the eight loci were down-regulated; while HMGA2 harbors a validated SOX10 response element (Fig. 6b), depletion of SOX10 activity did not reduce Hmga 2 expression. Further analysis will be required to determine if the SOX10 response element at Hmga2 regulates an adjacent locus or if depletion of SOX10 at specific developmental timepoints results in reduced Hmga 2 expression. Consistent with our findings, previous global analyses of SOX10 function revealed that two of the above eight loci are downstream of SOX10: Id2 and Notch1 [14]; our analysis now localizes at least some of the SOX10-dependent enhancers responsible for the regulation of these two loci.

SOX5 and SOX6 are members of the SOXD family of transcription factors and act as negative regulators of myelination in the central nervous system [38]; these proteins, which do not have transactivation or transrepression domains [39], inhibit the expression of SOX10 target genes (e.g., $M B P$ ) in oligodendrocytes by competing with SOX10 for DNA binding at sites within cis-acting regulatory elements. To allow oligodendrocyte differentiation and myelin production, SOX6 mRNA is targeted for degradation by two microRNAs (miR) in these cells: miR219 and miR-338 [40]. It was recently reported that SOX13 (the third and final member of the SOXD subgroup) also has an antagonistic effect on the ability of SOX10 to activate the expression of myelin genes in the central nervous system [41]. Indeed, SOX13 is among the group of 191 loci at which we identified a highly confident SOX10 binding site: a single, conserved genomic segment within SOX10 ChIP-seq and DNase-seq peaks $\sim 62 \mathrm{~kb}$ upstream of Sox13 (rn5 coordinates chr13:5542548655425636) was identified (Additional file 9). Interestingly, a relationship between SOXD and SOXE (SOX8, SOX9, and SOX10) transcription factors has been proposed since ablation of SOX8 or SOX9 (but not SOX10) 
reduces Sox6, but not Sox5, expression in the developing spinal cord [38].

In addition to genes encoding SOXD proteins, our studies predict that NOTCH1, HES1, MYCN, ID2, and ID4 are SOX10 target genes. NOTCH1 is a transmembrane receptor that regulates Schwann cell proliferation and inhibits Schwann cell differentiation in perinatal nerves, and facilitates dedifferentiation of Schwann cells after nerve injury [42]. HES1 is an effector of NOTCH signaling, acts as a transcriptional repressor [43, 44], and is highly expressed during early stages of Schwann cell development [42]. In cultured mouse oligodendrocytes, HES1 maintains cells in an immature state and overexpression of HES1 results in reduced expression of myelin related genes ( $M b p$ and $P l p)$ [45]. MYCN is a protooncogene and is known to inhibit astrocyte differentiation from neural precursor cells [46]; however, the role of MYCN during Schwann cell myelination has not been studied. Inhibitors of differentiation 2 and 4 (ID2 and ID4) are known to inhibit oligodendrocyte differentiation and the lack of both proteins results in premature oligodendrocyte differentiation [47-49]. Furthermore, Id 2 and Id4 expression declines in Schwann cell development and ID2 limits induction of myelin protein zero expression in primary Schwann cells [50, 51]. Consistent with our findings, RNA-seq of oligodendrocytes isolated at various stages of mouse brain development [52] show that Sox5, Sox6, Notch1, Hes1, Mycn, Id2, and Id4 are developmentally regulated in the central nervous system-Hmga2 does not appear to be expressed in the cells assessed in that study. Therefore, these genes likely play a role in preventing premature glial cell differentiation in both the central and peripheral nervous systems.

Combined with previous findings, our data predict a model (Fig. 7) where SOX10 activates the expression of genes that inhibit Schwann cell differentiation, possibly during early stages of Schwann cell development, thus preventing the precocious expression of myelin proteins. Subsequently, EGR2, NAB, and microRNAs are known to inhibit the expression of the negative regulators of myelination (e.g., SOXD proteins), which would allow the expression of myelin proteins. In addition to the data presented in this study, previous reports support specific aspects of this model. For example, EGR2 likely represses the expression of many of the eight loci reported here. EGR2 and NAB repress ID2 and ID4 before myelination via NAB binding to CHD4 [51] [conditional ablation of CHD4 in Schwann cells causes increased expression of immature Schwann cell genes including ID2 and delayed myelination, radial sorting defects, hypomyelination, and the persistence of promyelinating Schwann cells in conditional knockout mice [53]]. Furthermore, a comparison of SOX10 and EGR2 binding

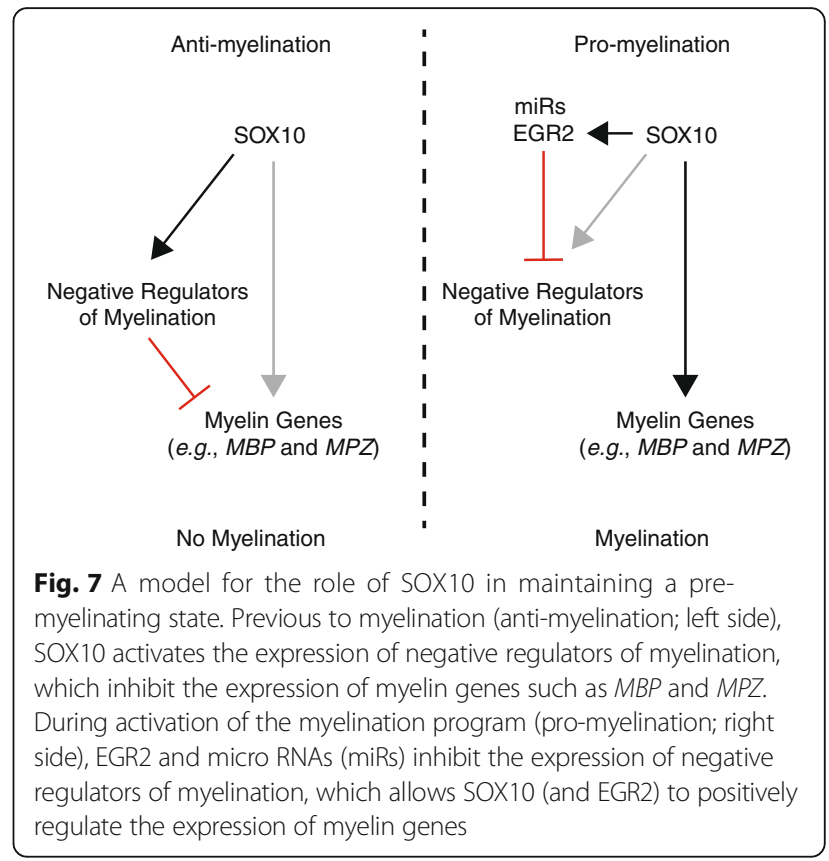

with expression profiles in Schwann cells treated with siRNA for SOX10 and EGR2-deficent peripheral nerves [14] revealed that NOTCH1 and $I D 2$ are SOX10-activated and EGR2-repressed, and ID2, HMGA2, SOX5, and ID4 remain high in peripheral nerves from Egr2- or Nabdeficient mice [51, 54]. Finally, SOX10 directly regulates the expression of EGR2 [28] and miR-338 [20]. In sum, our findings suggest that SOX10 has a role in maintaining a premyelinating state during non-myelinating stages of Schwann cell development.

\section{Conclusions}

In this study, we developed a computational and functional pipeline to expand the panel of known SOX10 response elements and target loci. These efforts predict a novel function for SOX10 in repressing myelination in early stages of Schwann cell (and possibly neural crest) development. Furthermore, we provide useful datasets for the scientific community and expand the mutational screening space for disease-causing mutations-or modifiers of disease-in patients with peripheral neuropathy and other SOX10-related phenotypes.

\section{Methods}

Computational identification and prioritization of SOX10 consensus sequences

To identify all SOX10 consensus sequences in the human genome we downloaded individual text files for each human chromosome (hg18) from the UCSC Human Genome Browser [30] and wrote a Perl script (available upon request) that examines each file for the consensus sequences (using a regular expression analysis) 'ACACA' or 
'ACAAD'; where ' $\mathrm{D}$ ' is a G, T, or A nucleotide. To identify two SOX10 consensus sequence monomers that are oriented in a head-to-head manner (and that may represent a dimeric SOX10 binding site) we wrote a second Perl script that examines the human chromosome text files and reports each 'ACACA' or 'ACAAD' consensus sequence that is five to 10 base pairs 5 ' to the reverse complement of this consensus sequence: 'TGTGT' or 'HTTGT', where ' $\mathrm{H}$ ' represents an A, C, or T nucleotide.

To identify genomic sequences that are identical between human, mouse, and chicken we downloaded the vertebrate (44 species) multiz alignment (maf) files from the UCSC Human Genome Browser (hg18) and extracted the alignments for human, mouse, and chicken. Next, we utilized the program ExactPlus [55] to identify all human sequences that are at least 5 base pairs long and identical between all three species. All subsequent computational analyses that assess for overlap between these and other datasets were performed using the UCSC Table Browser [56] and the 'intersection' tool. For these analyses we employed UCSC Genome custom tracks containing each: (1) human RefSeq (hg18) protein-coding sequence to exclude coding sequences; (2) human RefSeq entry (hg18) plus 2.5 kb upstream and $2.5 \mathrm{~kb}$ downstream of the transcriptional unit to identify regions that map to known genes; (3) SOX10 ChIP-seq peak in the rat genome (rn5) using HOMER analysis [57] (Additional file 11) of previously described P15 sciatic nerve data sets; and (4) DNase-seq peak in the rat genome (rn5) that has an F-Seq [58] score of at least 0.08 (see below).

To identify the 57 loci with a known or predicted role in peripheral nerve myelination we performed the following PubMed searches in September 2014: (1) each gene name plus 'Schwann'; and (2) each gene name plus 'Myelin'. We also searched for each gene name plus 'Schwannoma' in the GEO Profiles database at NCBI to determine if gene expression is depleted upon treatment with SOX10 siRNA [32].

\section{Luciferase reporter gene expression constructs}

Primers containing attB1 and attB2 Gateway cloning (Invitrogen) sequences were designed for each region identified by our computational pipeline (human genespecific primer sequences are provided in Additional file 5; rat gene-specific primer sequences are available upon request). Regions were PCR-amplified using human or rat genomic DNA and subsequently cloned into pDONR221 using BP clonase (Invitrogen) according to the manufacturer's instructions. The pDONR221 constructs were sequence verified and cloned upstream of an E1B minimal promoter [55] directing luciferase expression in the forward (pE1B Forward) or reverse (pE1B Reverse) orientation using LR clonase (Invitrogen) according to the manufacturer's instructions. Mutagenic primers to delete SOX10 consensus sequences (available upon request) were designed and site directed mutagenesis performed using the QuikChange II Mutagenesis Kit (Agilent Technologies) according to the manufacturer's instructions. The resulting mutant constructs were sequence verified to ensure that the SOX10 consensus site was deleted and that no other mutations were generated. Subsequently, the constructs were recombined into the $\mathrm{pE} 1 \mathrm{~B}$ luciferase vector as described above.

\section{Cell culture and luciferase assays}

Cultured rat Schwann cells (S16) [21, 22] were obtained ten years ago from Richard Quarles (NIH/NINDS) and mouse motor neuron derived cells (MN1) [23] were obtained 12 years ago from Kurt Fischbeck (NIH/NINDS). Both cell types were maintained in Dulbecco's modified Eagle's medium supplemented with $10 \%$ fetal bovine serum (Gibco) and $2 \mathrm{mM} \mathrm{L-glutamine} \mathrm{(Gibco).} \mathrm{For}$ luciferase assays, $1 \times 10^{4}$ cells were plated in each well of a 96-well culture plate (Corning Life Sciences) and incubated overnight at $37{ }^{\circ} \mathrm{C}$ in $5 \% \mathrm{CO}_{2}$. Cells were transfected using Lipofectamine 2000 (Invitrogen) according to the manufacturer's instructions. Each well was transfected with $200 \mathrm{ng}$ of the pE1B construct [55] and $2 \mathrm{ng}$ of pCMV-Renilla (to correct for experimental variation caused due to cell viability and transfection efficiency) in $50 \mathrm{uL}$ of Opti-MEM (Gibco) and $0.25 \mathrm{uL}$ Lipofectamine 2000. Cells were incubated at $37^{\circ} \mathrm{C}$ for $4 \mathrm{~h}$ and then grown in standard growth media for $48 \mathrm{~h}$. For overexpression studies, $100 \mathrm{ng}$ of a construct to express wildtype or E189X SOX10 [8], wild-type SOX8, SOX9, or EGR2 was included in the transfection reaction. $48 \mathrm{~h}$ after transfection the cells were lysed in $20 \mathrm{uL}$ of $1 \mathrm{X}$ passive lysis buffer (Promega) for $1 \mathrm{~h} .10 \mathrm{uL}$ of the lysate was transferred to a 96-well assay plate (Corning Life Sciences) and a Dual Luciferase Assay (Promega) was performed to determine the activities of luciferase and renilla. Luciferase activity was normalized to renilla activity and the fold increase in luciferase activity was calculated relative to the empty control vector, which does not contain a genomic insert. For each genomic segment, three independently isolated reporter gene constructs were studied in eight technical replicates for a total of 24 reactions per segment. Statistical calculations comparing the regulatory activity of different alleles were performed using the Mann-Whitney-Wilcoxon test.

\section{Standard and quantitative RT-PCR}

Total RNA was isolated from S16 and MN1 cells. $1 \times 10^{5}$ cells were plated in a 6-well plate and incubated overnight at $37{ }^{\circ} \mathrm{C}$ in $5 \% \mathrm{CO}_{2}$. Each well was transfected using 4 ug of wild-type or E189X SOX10 [8] in $1 \mathrm{~mL}$ of Opti-MEM and $10 \mathrm{uL}$ of Lipofectamine 2000. Mock 
transfections were performed in the absence of DNA. Cells were incubated at $37{ }^{\circ} \mathrm{C}$ for $4 \mathrm{~h}$ and then grown in standard media. After $72 \mathrm{~h}$, total RNA was isolated from the transfected cells using the RNeasy kit (Qiagen). Subsequently, cDNA was synthesized using 1 ug of total RNA and the High Capacity cDNA Reverse Transcription Kit (Life Technologies) according to manufacturer's instructions. RT-PCR was performed on isolated cDNA using gene specific primers (sequences available upon request). A PCR for $\beta$-actin served as a positive control. All PCR products were subjected to DNA sequencing to confirm specificity. RNA was purified from three independent rat sciatic nerves at the P1, P15, and adult timepoints using the Qiagen RNeasy Lipid kit, and quantitative RT-PCR was performed as described [20].

\section{5' Rapid amplification of CDNA ends}

First strand cDNA libraries were synthesized using total RNA isolated from S16 cells and a primer designed within exon 5 of Sox6. The cDNA was TdT-tailed using the 5'RACE System (Invitrogen). Subsequently, two nested PCRs were performed: one using a reverse primer designed within exon 4 of Sox6 and a second using a reverse primer designed within exon 3 of Sox6 (primer sequences available upon request). The nested PCR products were separated on a $1 \%$ agarose gel, excised, and purified using the QIAquick gel extraction kit (Qiagen). Gel purified PCR products were TA cloned (Invitrogen) according to manufacturers instructions and 48 clones were subjected to Sanger sequencing: 44 of the resulting sequences correctly mapped to the rat Sox6 locus.

\section{siRNA-mediated depletion of SOX10}

Control siRNA (siControl 1, Ambion catalog number AM4611) or Sox10 siRNA (siSox10 1, Life Technologies catalog number s131239) were transfected into S16 cells as described using the Amaxa Nucleofection system following the manufacturer's instructions. At $48 \mathrm{~h}$ post-transfection, RNA was isolated using Tri-Reagent (Ambion) and analyzed by quantitative RT-PCR as described [20].

\section{DNase hypersensitivity site identification}

DNase-seq was performed with three biological replicates of rat Schwann (S16) cells at passage numbers five, eight, and 14. Each replicate contained $\sim 20$ million cells frozen into $1 \mathrm{ml}$ of freezing media. Cells were thawed and DNase-seq libraries generated as previously described [59] with the exception of adding a 5' phosphate to linker 1 to increase the ligation efficiency. DNase-seq libraries from three replicates were pooled into one lane of an Illumina Hi-Seq 2000. Raw reads were aligned to the rat (rn5) genome using Bowtie [60] and unique mapping with up to two mismatches allowed in an alignment. For the three samples, $69.2 \%(36,295,401)$, $70.8 \%(43,564,606)$, and $67.9 \%(39,579,719)$ of the reads mapped to rn5. Peaks were called using F-Seq and the default settings [58]. For the three samples: 502,787 (sample 1), 438,254 (sample 2), and 412,267 (sample 3) peaks were identified. 149,342 were shared among all three samples (Additional file 12). We next used sample 2 as a representative experiment and extracted all DNase-seq peaks with an F-Seq score [52] of at least 0.08 . This revealed a set of 31,845 peaks (7.3\%; Additional file 13) that were used to prioritize SOX10 response elements (see section 'Computational identification and prioritization of SOX10 consensus sequences' above). Data from the three samples have been submitted to GEO (GSM2166058, GSM2166059 and GSM2166060).

\section{Additional files}

Additional file 1: Monomeric SOX10 consensus sequences in the human genome provided as a UCSC Human Genome Browser (hg18) custom track. The fourth column ('name') shows the predicted SOX10 binding site sequence. (ZIP $222208 \mathrm{~kb}$ )

Additional file 2: Dimeric SOX10 consensus sequences with an intervening sequence of five to 10 base pairs in the human genome provided as a UCSC Human Genome Browser (hg18) custom track. The fourth column ('name') shows the predicted SOX10 binding site sequence. (TXT $22732 \mathrm{~kb}$ )

Additional file 3: Genomic segments at least five base pairs long and conserved between human, mouse, and chicken provided as a UCSC Human Genome Browser (hg18) custom track. The fourth column ('name') shows the position and length of the conserved segment. (TXT 87756 kb)

Additional file 4: Genomic coordinates (hg18; UCSC Human Genome Browser) of 238 SOX 10 consensus sequences that are conserved between human, mouse, and chicken and that map to non-coding sequences near a RefSeq gene. Columns are labeled in the file. (XLSX 29 kb)

Additional file 5: Genomic coordinates (hg18; UCSC Human Genome Browser) and primer sequences for 57 genomic segments studied in luciferase assays presented in Fig. 1. Columns are labeled in the file. (XLSX $44 \mathrm{~kb}$ )

Additional file 6: Figure S1. Three genomic regions display orientation-specific regulatory activity in motor neurons. The seven active regions from Fig. 1 were tested in forward (grey bars) and reverse (white bars) orientation in mouse motor neuron (MN1) cells. Luciferase data are expressed relative to a control vector without a genomic segment ('Empty'). The scale of the $y$-axis is the same as in Fig. 2 to allow comparisons. Error bars indicate standard deviations. (PDF 97 kb)

Additional file 7: Figure S2. SOX8 and SOX9 also increase the regulatory activity of SOX10-CCS-13, SOX10-CCS-19, and SOX10-CCS-51. Luciferase reporter gene constructs harboring SOX10-CCS-13, SOX10-CCS-19, or SOX10-CCS-51 were transfected into mouse motor neurons (MN1) with constructs to express SOX8 or SOX9. The luciferase activity associated with each construct in the presence of SOX8 or SOX9 is expressed relative to that of the same construct in the absence of these transcription factors. Error bars indicate standard deviations. Please note that the SOX10 data are identical to those in Fig. 3a and are included to facilitate a comparison. (PDF 330 kb)

Additional file 8: Figure S3. EGR2 does not act synergistically with SOX10 to activate SOX10-CCS-13, SOX10-CCS-19, or SOX10-CCS-51 in vitro. Luciferase reporter gene constructs harboring SOX10-CCS-13, SOX10-CCS-19, or SOX10-CCS-51 were transfected into mouse motor neurons (MN1) with constructs to express EGR2 and/or SOX10. The luciferase activity associated with each construct in the presence of the 
transcription factor(s) is expressed relative to that of the untreated reporter construct. Error bars indicate standard deviations. Please note that the SOX10 data are identical to those in Fig. 3a and are included to facilitate a comparison. (PDF $328 \mathrm{~kb}$ )

Additional file 9: Genomic coordinates and custom tracks (rn5; UCSC Rat Genome Browser) for SOX10 ChIP-seq peaks that map to both a DNase hypersensitivity site and at least one SOX10 monomeric consensus sequence that is conserved between human, mouse, and chicken. Columns are labeled in the file. (XLSX $23 \mathrm{~kb})$

Additional file 10: Figure S4. SOX10 regulates genes that inhibit glial cell differentiation in vivo. Primary Schwann cells were extracted and grown from three independent rat adult sciatic nerves. Cells were treated with a control siRNA or an siRNA targeted against Sox10 as in Fig. 7c. Quantitative RT-PCR was used to measure expression levels of each indicated gene. The effect on expression of each gene (indicated across the bottom) is expressed relative to the control siRNA and error bars indicate standard deviations. Please note that, consistent with the in vitro data, Hmga2 did not show a decrease in expression levels in vivo (data not shown). (PDF $334 \mathrm{~kb}$ )

Additional file 11: SOX10 ChIP-seq peaks in the rat genome using HOMER analysis. The data are provided as a UCSC Rat Genome Browser (rn5) custom track. (TXT $1526 \mathrm{~kb}$ )

Additional file 12: DNase-seq peaks identified in $\mathrm{S} 16$ cells that are common to all three experimental replicates. The data are provided as a UCSC Rat Genome Browser (rn5) custom track and the fourth column ('name') shows the F-Seq score for the peak. (TXT $5171 \mathrm{~kb}$ )

Additional file 13: DNase-seq peaks identified in $\mathrm{S16}$ cells from experimental sample 2 that have an F-Seq score of at least 0.08. The data are provided as a UCSC Rat Genome Browser (rn5) custom track and the fourth column ('name') shows the F-Seq score for the peak. (TXT 1075 kb)

\section{Abbreviations}

CCS: Conserved Consensus Sequence; ChIP-seq: Chromatin immunoprecipitation followed by sequencing; DNase-seq: DNase I hypersensitivity sites followed by sequencing; MCS: Multiple species Conserved Sequence; PCR: Polymerase Chain Reaction; PNS: Peripheral Nervous System; SOX10: Sry-box 10

\section{Acknowledgements}

The authors would like to thank Ken Kwan, Ken Inoue, and Jim Lupski for SOX protein expression constructs; Jeff Kidd for assisting with data organization and storage; Weisheng Wu from the University of Michigan Bioinformatics Core for assisting with DNase-seq data alignment and peak calling; and Camila Lopez-Anido for critical evaluation of the manuscript.

\section{Funding}

This project was funded by the National Institute of Neurological Diseases and Stroke (NS073748 to A.A. and NS083841 to J.S.) and the Intramural Research Program of the National Human Genome Research Institute. C.G. was supported by funds from the Cellular and Molecular Biology Program at the University of Michigan. W.D.L. was supported by the National Institutes of Health Genetics Training Grant (GM007544) and an EDGE Award from the Endowment for the Basic Sciences at the University of Michigan. J.F.R-.M. was supported by a National Science Foundation Pre-Doctoral Fellowship.

\section{Availability of data and materials}

All reagents will be available upon request to the corresponding author. All bioinformatic and genome-wide functional data have been provided as additional material (see below).

\section{Authors' contributions}

WDL and AA wrote Perl scripts to identify SOX10 binding sites; JFR-M and JS designed and performed qRT-PCR experiments; ABP and JCM designed and wrote the ExactPlus program to examine multiple-species alignments; LS and $G C$ designed and performed DNase-seq experiments. CG, WDL, and AA designed, performed and analyzed all of the remaining data; $C G, W D L, J S$, and AA wrote the manuscript. All authors read and approved the manuscript.

\section{Competing interests}

The authors in this study declare no competing interests.

\section{Consent for publication}

Not applicable.

\section{Ethics approval}

All experiments involving animals were performed by the Svaren laboratory at the University of Wisconsin. As such, these studies followed experimental protocols approved by the University of Wisconsin School of Veterinary Medicine Institutional Animal Care and Use Committee.

\section{Author details}

${ }^{1}$ Cellular and Molecular Biology Program, University of Michigan Medical School, Ann Arbor, Ml 48109, USA. '2Department of Human Genetics, University of Michigan Medical School, Ann Arbor, MI 48109, USA. ${ }^{3}$ Cellular and Molecular Pathology Program, University of Wisconsin-Madison, Madison, WI 53706, USA. ${ }^{4}$ Genome Technology Branch, National Human Genome Research Institute, National Institutes of Health, Bethesda, MD 20892, USA. ${ }^{5}$ Center for Genomic and Computational Biology, Duke University Medical Center, Durham, NC 27708, USA. ${ }^{6}$ Department of Pediatrics, Duke University Medical Center, Durham, NC 27708, USA. ${ }^{7}$ Waisman Center, University of Wisconsin-Madison, Madison, WI 53706, USA. ${ }^{8}$ Department of Comparative Biosciences, University of Wisconsin-Madison, Madison, WI 53706, USA. 'Department of Neurology, University of Michigan Medical School, Ann Arbor, Ml 48109, USA

Received: 20 April 2016 Accepted: 18 October 2016

Published online: 07 November 2016

\section{References}

1. Jessen KR, Mirsky R. The origin and development of glial cells in peripheral nerves. Nat Rev Neurosci. 2005;6:671-82

2. Stolt CC, Wegner M. Schwann cells and their transcriptional network: Evolution of key regulators of peripheral myelination. Brain Res. 2016; 1641:101-10.

3. Kuhlbrodt K, Herbarth B, Sock E, Hermans-Borgmeyer I, Wegner M. Sox10, a novel transcriptional modulator in glial cells. J Neurosci. 1998; 18:237-50.

4. Britsch S, Goerich DE, Riethmacher D, Peirano RI, Rossner M, Nave KA, et al. The transcription factor Sox10 is a key regulator of peripheral glial development. Genes Dev. 2001;15:66-78.

5. Finzsch M, Schreiner S, Kichko T, Reeh P, Tamm ER, Bösl MR, et al. Sox10 is required for Schwann cell identity and progression beyond the immature Schwann cell stage. J Cell Biol. 2010;189:701-12.

6. Bremer M, Fröb F, Kichko T, Reeh P, Tamm ER, Suter U, et al. Sox10 is required for Schwann-cell homeostasis and myelin maintenance in the adult peripheral nerve. Glia. 2011;59:1022-32.

7. Inoue K, Tanabe Y, Lupski JR. Myelin deficiencies in both the central and the peripheral nervous systems associated with a SOX10 mutation. Ann Neurol. 1999:46:313-8.

8. Inoue K, Khajavi M, Ohyama T, Hirabayashi S-I, Wilson J, Reggin JD, et al. Molecular mechanism for distinct neurological phenotypes conveyed by allelic truncating mutations. Nat Genet. 2004;36:361.

9. Bondurand N, Girard M, Pingault V, Lemort N, Dubourg O, Goossens M. Human Connexin 32, a gap junction protein altered in the X-linked form of Charcot-Marie-Tooth disease, is directly regulated by the transcription factor SOX10. Hum Mol Genet. 2001:10:2783-95.

10. Jones EA, Lopez-Anido C, Srinivasan R, Krueger C, Chang L-W, Nagarajan R, et al. Regulation of the PMP22 gene through an intronic enhancer. J Neurosci. 2011;31:4242-50

11. Jones EA, Jang S-W, Mager GM, Chang L-W, Srinivasan R, Gokey NG, et al. Interactions of Sox10 and Egr2 in myelin gene regulation. Neuron Glia Biol. 2007:3:377-87.

12. LeBlanc SE, Jang S-W, Ward RM, Wrabetz $L$, Svaren J. Direct regulation of myelin protein zero expression by the Egr2 transactivator. J Biol Chem. 2006:281:5453-60.

13. Peirano Rl, Goerich DE, Riethmacher D, Wegner M. Protein zero gene expression is regulated by the glial transcription factor Sox10. Mol Cell Biol. 2000; 20:3198-209 
14. Srinivasan R, Sun G, Keles S, Jones EA, Jang S-W, Krueger C, et al. Genomewide analysis of EGR2/SOX10 binding in myelinating peripheral nerve. Nucleic Acids Res. 2012;40:6449-60.

15. Lopez-Anido C, Sun G, Koenning M, Srinivasan R, Hung HA, Emery B, et al. Differential Sox10 genomic occupancy in myelinating glia. Glia. 2015;63:1897-914.

16. Peirano RI, Wegner M. The glial transcription factor Sox10 binds to DNA both as monomer and dimer with different functional consequences. Nucleic Acids Res. 2000;28:3047-55.

17. Antonellis A, Green ED. Inter-Species Comparative Sequence Analysis: Applications in Human Disease Research and Genomic Medicine. In: Willard HF, Ginsburg GS, editors. Genomic and Personalized Medicine. Salt Lake City: Academic; 2008. p. 120-30.

18. Antonellis A, Huynh JL, Lee-Lin S-Q, Vinton RM, Renaud G, Loftus SK, et al. Identification of neural crest and glial enhancers at the mouse Sox10 locus through transgenesis in zebrafish. PLoS Genet. 2008;4:e1000174.

19. Hodonsky CJ, Kleinbrink EL, Charney KN, Prasad M, Bessling SL, Jones EA, et al. SOX10 regulates expression of the SH3-domain kinase binding protein 1 (Sh3kbp1) locus in Schwann cells via an alternative promoter. Mol Cell Neurosci. 2012;49:85-96

20. Gokey NG, Srinivasan R, Lopez-Anido C, Krueger C, Svaren J. Developmental regulation of microRNA expression in Schwann cells. Mol Cell Biol. 2012:32:558-68.

21. Goda S, Hammer J, Kobiler D, Quarles RH. Expression of the myelinassociated glycoprotein in cultures of immortalized Schwann cells. J Neurochem. 1991;56:1354-61.

22. Toda K, Small JA, Goda S, Quarles RH. Biochemical and cellular properties of three immortalized Schwann cell lines expressing different levels of the myelin-associated glycoprotein. J Neurochem. 1994;63:1646-57.

23. Salazar-Grueso EF, Kim S, Kim H. Embryonic mouse spinal cord motor neuron hybrid cells. Neuroreport. 1991;2:505-8.

24. Stolt CC, Wegner M. SoxE function in vertebrate nervous system development Int J Biochem Cell Biol. 2010;42:437-40.

25. Fogarty EA, Brewer MH, Rodriguez-Molina JF, Law WD, Ma KH, Steinberg NM, et al. SOX10 Regulates an Alternative Promoter at the Charcot-MarieTooth Disease Locus MTMR2. Hum Mol Genet. 2016;00:1-12.

26. Emery B. Playing the field: Sox 10 recruits different partners to drive central and peripheral myelination. PLoS Genet. 2013;9:e1003918.

27. Harris ML, Baxter LL, Loftus SK, Pavan WJ. Sox proteins in melanocyte development and melanoma. Pigment Cell Melanoma Res. 2010;23:496-513.

28. Ghislain J, Charnay P. Control of myelination in Schwann cells: a Krox20 cis-regulatory element integrates Oct6, Brn2 and Sox10 activities. EMBO Rep. 2006;7:52-8.

29. Brewer MH, Ma KH, Beecham GW, Gopinath C, the Inherited Neuropathy Consortium (INC), Baas F, et al. Haplotype-Specific Modulation of a SOX10/ CREB Response Element at the Charcot-Marie-Tooth Disease Type 4C Locus SH3TC2. Hum Mol Genet. 2014;23:5171-87.

30. Kent WJ, Sugnet CW, Furey TS, Roskin KM. The human genome browser at UCSC. Genome Res. 2002;12:996-1006.

31. Jones EA, Brewer MH, Srinivasan R, Krueger C, Sun G, Charney KN, et al. Distal enhancers upstream of the Charcot-Marie-Tooth type 1A disease gene PMP22. Hum Mol Genet. 2012;21:1581-91.

32. Lee KE, Nam S, Cho E-A, Seong I, Limb J-K, Lee S, et al. Identification of direct regulatory targets of the transcription factor Sox 10 based on function and conservation. BMC Genomics. 2008;9:408.

33. Kwasnieski JC, Fiore C, Chaudhari HG, Cohen BA. High-throughput functional testing of ENCODE segmentation predictions. Genome Res. 2014;24:1595-602.

34. Kelsh RN. Sorting out Sox10 functions in neural crest development. Bioessays. 2006;28:788-98.

35. Hai M, Muja N, DeVries GH, Quarles RH, Patel PI. Comparative analysis of Schwann cell lines as model systems for myelin gene transcription studies. J Neurosci Res. 2002;69:497-508.

36. Wei Q, Miskimins WK, Miskimins R. Sox10 acts as a tissue-specific transcription factor enhancing activation of the myelin basic protein gene promoter by p27Kip1 and Sp1. J Neurosci Res. 2004;78:796-802.

37. Li H, Lu Y, Smith HK, Richardson WD. Olig1 and Sox 10 interact synergistically to drive myelin basic protein transcription in oligodendrocytes. J Neurosci. 2007; 27:14375-82.

38. Stolt CC, Schlierf A, Lommes P, Hillgärtner S, Werner T, Kosian T, et al. SoxD proteins influence multiple stages of oligodendrocyte development and modulate SoxE protein function. Dev Cell. 2006;11:697-709.

39. Lefebvre $V$. The SoxD transcription factors-Sox5, Sox6, and Sox13-are key cell fate modulators. Int J Biochem Cell Biol. 2010;42:429-32.
40. Zhao X, He X, Han X, Yu Y, Ye F, Chen Y, et al. MicroRNA-mediated control of oligodendrocyte differentiation. Neuron. 2010;65:612-26.

41. Baroti $T$, Schillinger $A$, Wegner M. Sox 13 functionally complements the related Sox5 and Sox6 as important developmental modulators in mouse spinal cord oligodendrocytes. J Neurochem. 2015;136:316-28.

42. Woodhoo A, Alonso MBD, Droggiti A, Turmaine M, D'Antonio M, Parkinson DB, et al. Notch controls embryonic Schwann cell differentiation, postnatal myelination and adult plasticity. Nat Neurosci. 2009;12:839-47.

43. Jarriault S, Brou C, Logeat F, Schroeter EH, Kopan R, Israel A. Signalling downstream of activated mammalian Notch. Nature. 1995;377:355-8.

44. Sasai $Y$, Kageyama R, Tagawa $Y$, Shigemoto R, Nakanishi S. Two mammalian helix-loop-helix factors structurally related to Drosophila hairy and Enhancer of split. Genes Dev. 1992;6:2620-34.

45. Ogata T, Ueno T, Hoshikawa S, Ito J, Okazaki R, Hayakawa K, et al. Hes 1 functions downstream of growth factors to maintain oligodendrocyte lineage cells in the early progenitor stage. Neuroscience. 2011;176:132-41.

46. Sanosaka T, Namihira M, Asano H, Kohyama J, Aisaki K, Igarashi K, et al. Identification of genes that restrict astrocyte differentiation of midgestational neural precursor cells. Neuroscience. 2008;155:780-8.

47. Marin-Husstege M, He Y, Li J, Kondo T, Sablitzky F, Casaccia-Bonnefil P. Multiple roles of Id4 in developmental myelination: predicted outcomes and unexpected findings. Glia. 2006:54:285-96.

48. Kondo T, Raff M. The Id4 HLH protein and the timing of oligodendrocyte differentiation. EMBO J. 2000;19:1998-2007.

49. Wang S, Sdrulla A, Johnson JE, Yokota Y, Barres BA. A role for the helix-loophelix protein Id2 in the control of oligodendrocyte development. Neuron. 2001;29:603-14.

50. Stewart HJ, Zoidl G, Rossner M, Brennan A, Zoidl C, Nave KA, et al. Helixloop-helix proteins in Schwann cells: a study of regulation and subcellular localization of Ids, REB, and E12/47 during embryonic and postnatal development. J Neurosci Res. 1997;50:684-701.

51. Mager GM, Ward RM, Srinivasan R, Jang S-W, Wrabetz L, Svaren J. Active gene repression by the Egr2.NAB complex during peripheral nerve myelination. J Biol Chem. 2008;283:18187-97.

52. Zhang Y, Chen K, Sloan SA, Bennett ML, Scholze AR, O'Keeffe S, et al. An RNA-sequencing transcriptome and splicing database of glia, neurons, and vascular cells of the cerebral cortex. J Neurosci. 2014:34:11929-47.

53. Hung H, Kohnken R, Svaren J. The nucleosome remodeling and deacetylase chromatin remodeling (NuRD) complex is required for peripheral nerve myelination. J Neurosci. 2012;32:1517-27.

54. Le N, Nagarajan R, Wang JYT, Svaren J, LaPash C, Araki T, et al. Nab proteins are essential for peripheral nervous system myelination. Nat Neurosci. 2005; 8:932-40.

55. Antonellis A, Bennett WR, Menheniott TR, Prasad AB, Lee-Lin S-Q, NISC Comparative Sequencing Program, et al. Deletion of long-range sequences at Sox10 compromises developmental expression in a mouse model of Waardenburg-Shah (WS4) syndrome. Hum Mol Genet. 2006;15:259-71.

56. Karolchik D, Hinrichs AS, Furey TS. The UCSC Table Browser data retrieval tool. Nucleic Acids Res. 2004;32(Database issue):D493-6.

57. Heinz S, Benner C, Spann N, Bertolino E, Lin YC, Laslo P, et al. Simple combinations of lineage-determining transcription factors prime cis regulatory elements required for macrophage and $B$ cell identities. Mol Cell. 2010;38:576-89.

58. Boyle AP, Guinney J, Crawford GE, Furey TS. F-Seq: a feature density estimator for high-throughput sequence tags. Bioinformatics. 2008;24:2537-8.

59. Song L, Crawford GE. DNase-seq: a high-resolution technique for mapping active gene regulatory elements across the genome from mammalian cells. Cold Spring Harb Protoc. 2010;2010:pdb.prot5384.

60. Langmead B. Aligning short sequencing reads with Bowtie. Current protocols in bioinformatics. 2010 